\title{
PERFIL EPIDEMIOLÓGICO DA ANEMIA FERROPRIVA NO SERVIÇO DE HEMATOLOGIA DE UM HOSPITAL PÚBLICO, ESTADO DE SÃO PAULO, BRASIL
}

Kleber Jordão Souza, Vinicius de Freitas Tabox, Juliana Mussolini Celestino de Oliveira, Marcio Rossato Pierezan, Rogério Giuffrida, Rebeca Carvalho Bressa, José Antonio Nascimento Bressa

Universidade do Oeste Paulista - UNOESTE, Faculdade de Medicina, Presidente Prudente, SP.

Correspondência para: Kleber Jordão Souza - klehjs@hotmail.com

\section{RESUMO}

A anemia ferropriva possui diversas etiologias, como nutricional, absortivas, metabólicas ou distributivas, sangramento e aumento das necessidades fisiológicas. Apesar do impacto epidemiológico e clínico, a anemia ainda permanece incerta quanto à epidemiologia, principalmente pela falta de dados consistentes em nossa região. O objetivo deste estudo foi determinar o perfil epidemiológico dos pacientes atendidos pelo serviço de hematologia de um hospital público do estado de São Paulo no período de 2005 a 2010, com diagnóstico de anemia ferropriva. Trata-se de um estudo transversal que utilizou o prontuário dos pacientes, sendo avaliados: sexo, idade, exames laboratoriais e etiologia. A associação entre faixa etária, grupo populacional e sexo com as origens etiológicas da anemia ferropriva foi estudada com o teste de Qui-quadrado de Pearson e as estimativas das razões de chances (Odds-ratio) por ponto e por intervalos com 95\% de confiança. Foram incluídos 188 pacientes com diagnóstico de anemia ferropriva. Destes, 128 (68\%) eram do sexo feminino e 60 (32\%) do sexo masculino, a média etária geral foi de 52 anos e as etiologias mais prevalentes foram: perda sanguínea por via urogenital 71 $(37,70 \%)$, gastrointestinal alta $(34,57 \%)$ e baixa $(23,94 \%)$. O perfil dos pacientes com anemia ferropriva que procuraram o serviço de hematologia, mostrou que a menorragia constitui a principal etiologia para mulheres em idade reprodutiva, representando cerca de $1 / 3$ dos casos. As perdas gastrointestinais alta e baixa, representam mais da metade dos casos e o risco de chances, para ambas é maior para homens e idosos independente do sexo.

Palavras-chave: estudos transversais, epidemiologia, etiologia, anemia hipocrômica, anemia ferropriva.

\section{EPIDEMIOLOGY PROFILE OF IRON DEFICIENCY ANEMIA IN HEMATOLOGY SERVICE OF A PUBLIC HOSPITAL, SÃO PAULO STATE, BRAZIL}

\begin{abstract}
Iron deficiency anemia has many causes, as nutrition, absorptive, metabolic or distributive, bleeding and increased physiological needs. Although the epidemiological and clinical impact, anemia remains uncertain as to epidemiology, particularly the lack of consistent data in our region. The aim of this study was to determine the epidemiological profile of patients attended by the hematology service of a public hospital in the state of São Paulo in the period 2005-2010 with a diagnosis of iron deficiency anemia. This is a cross-sectional study that used records of patients being evaluated: sex, age, laboratory tests and etiology. The association between age, sex and population group with the etiological origins of iron deficiency anemia was studied with Pearson's Qui-square test and the estimates of the odds ratio point by point and by interval with a $95 \%$ confidence interval. Were included 188 patients diagnosed with iron deficiency anemia and, of whom 128 (68\%) were female and 60 patients (32\%) males, overall mean age of 52 years and the most prevalent etiologies were included were: blood loss via urogenital 71 (37.70\%), upper
\end{abstract}


gastrointestinal (34.57\%) and low (23.94\%). The profile of patients with iron deficiency anemia who sought the hematology service showed that menorrhagia is the main etiology for women of reproductive age, accounting for about one third of cases. Gastrointestinal losses high and low, represent more than half of the cases and the risk of chances for both is greater for men and elderly patients independent of sex.

Keywords: sectional studies, epidemiology, etiology, hypochromic anemia, iron deficiency anemia. 


\section{INTRODUÇÃO}

O ferro é vital para a homeostase celular, sendo componente da hemoglobina e mioglobina e tem funções diversas como transporte de oxigênio, síntese de DNA e metabolismo energético ${ }^{1}$. De acordo com Devuyst et al. ${ }^{2}$, as perdas fisiológicas do ferro são observadas na descamação do epitélio intestinal, no suor e por perdas menstruais. No homem, a perda diária é cerca de $1 \mathrm{mg}$ e na mulher, $2 \mathrm{mg}$ de ferro ${ }^{3}$. A absorção diária, regulada pela hepcidina e eritropoetina, repõe as perdas fisiológicas ${ }^{4}$. Entretanto, quando o balanço entre o ferro perdido e absorvido é negativo, ocorre a deficiência de ferro $^{5}$, que acarretará consequências para todo o organismo, sendo a anemia a manifestação mais relevante ${ }^{1}$.

A anemia ferropriva é de etiologia multifatorial. As causas podem ser alimentares, absortivas, metabólicas ou distributivas, perdas ou aumento das necessidades fisiológicas ${ }^{6}$. Apesar de apresentar ampla distribuição na população em geral e grande impacto clínico, os dados epidemiológicos a respeito das causas da anemia ainda são inconsistentes ${ }^{6,7}$.

O objetivo deste estudo foi determinar o perfil epidemiológico dos pacientes atendidos, com diagnóstico de anemia ferropriva, no serviço de hematologia de um hospital público do Estado de São Paulo.

\section{MÉTODOLOGIA}

Estudo transversal descritivo do perfil epidemiológico da anemia ferropriva, com ênfase no diagnóstico etiológico, e baseado nas informações dos prontuários dos pacientes atendidos pelo serviço de hematologia de um hospital público do Estado de São Paulo no período de 2005 a 2010.

Para inclusão no estudo, foram adotados os seguintes parâmetros: pessoas de ambos os sexos, com idade maior ou igual a 18 anos, valores de hemoglobina $<13 \mathrm{~g} / \mathrm{dL}$ e <12 g/dL, para homem e mulher, respectivamente, Hemoglobina Corpuscular Média $(\mathrm{HCM})<27 \quad \mathrm{pg}^{8}$, saturação de transferrina $<20 \%$ e concentração sérica de ferritina $<30 \mu \mathrm{g} / \mathrm{L}$, conforme algorítimo simplificado e traduzido (Figura 1$)^{9}$.

As seguintes informações foram coletadas dos prontuários: sexo (masculino ou feminino), idade (média e por faixa etária) e grupo populacional (adulto ou idoso, sendo categorizado como idoso os pacientes com idade maior ou igual a 65 anos), considerando a data do diagnóstico, exames laboratoriais pertinentes a anemia como hemoglobina, HCM, ferritina, saturação de 
transferrina, e a provável etiologia. Os pacientes foram divididos em cinco grupos: perdas de origem urogenital, perda gastrointestinal alta e baixa, origem gestacional/aumento do consumo de ferro e deficiência nutricional. Em cada grupo foram coletadas, a partir dos prontuários, informações com relação à presença de doenças ou intervenções cirúrgicas que originaram a anemia.

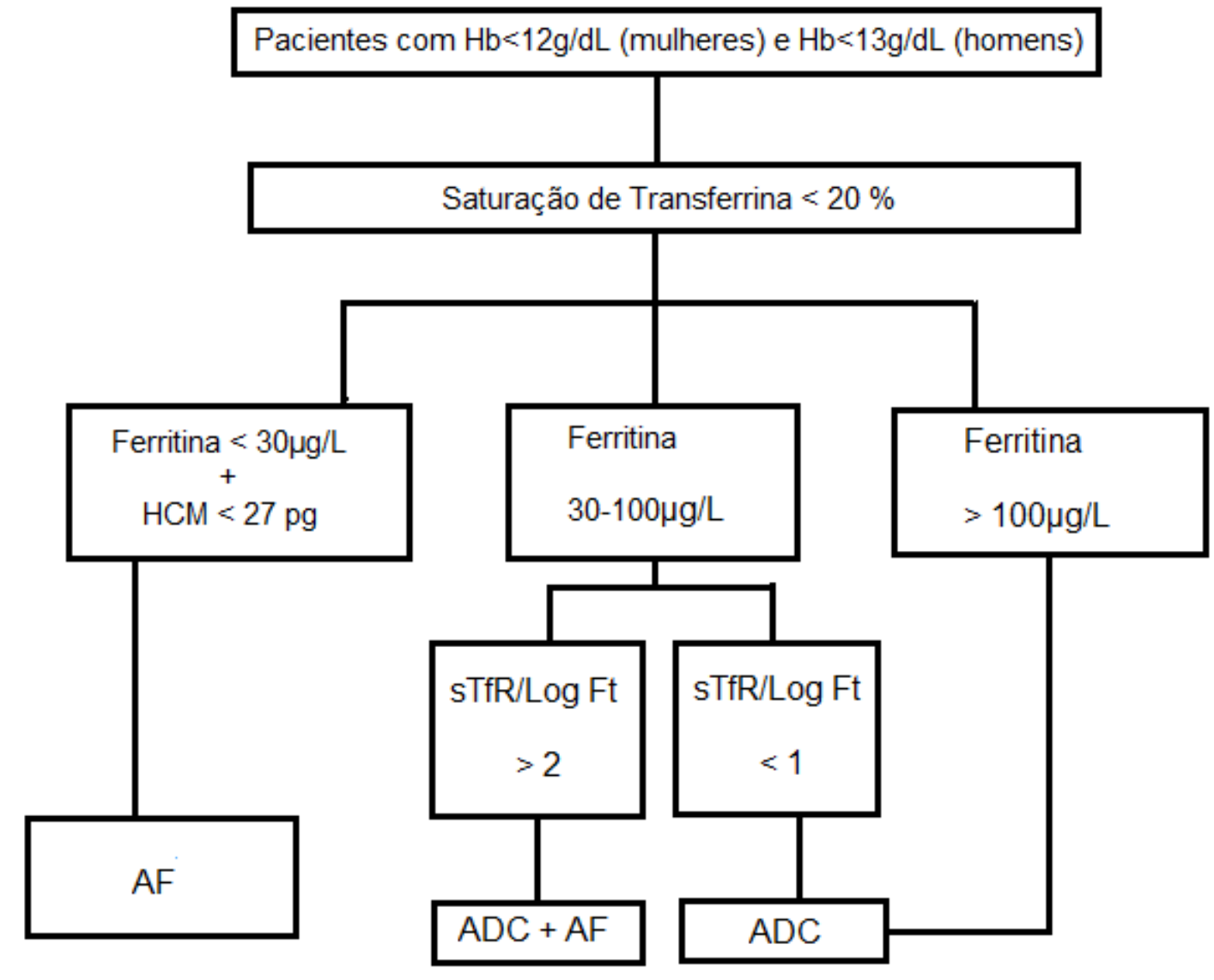

Legenda: Hb - Hemoglobina; HCM - Hemoglobina Corpuscular Média;sTfR - Solubilidade dos Receptores de Transferrina; Log Ft - Logarítimo da ferritina; AF - Anemia Ferropriva; ADC - Anemia de Doença Crônica

Figura 1. Algorítimo simplificado para o diagnóstico da anemia ferropriva ${ }^{9}$.

Foram excluídos os prontuários de pacientes com idade inferior a 18 anos, que não possuíam as variáveis pesquisadas, que possuíam outras etiologias concomitantes com características inflamatórias ou crônicas e, considerando que a ferritina é uma proteina que se eleva nessas situações ${ }^{5}$, foi considerado o corte em 30 da ferritina para inclusão neste trabalho de acordo com a Figura 1, que apesar de levar em conta a 
solubilidade da transferrina para o cálculo que diferencial em anemia de doença crônica ou anemia mista, não faz parte do foco deste trabalho e, portanto, não foi utilizado o fluxograma para os casos em que a ferritina foi superior ao corte.

Os pacientes que continham no prontuário informação sobre hemoglobinopatia foram também excluidos.

Os valores observados foram categorizados e sumarizados nas tabelas de frequência. Para estudar a associação entre faixa etária, grupo populacional e sexo com as origens etiológicas de anemia ferropriva, recorreu-se ao teste de Qui-quadrado de Pearson ou Exato de Fisher. As razões de chances (Odds-ratio) por ponto e por intervalos com $95 \%$ de confiança foram estimados por análise univariada. Para a variável faixa etária, as categorias foram avaliadas duas a duas, considerando-se como referência a primeira categoria. Todas as análises foram realizadas com o uso do software SPSS for Windows v.13.0. O nível de significância adotado para todas as análises foi de 5\%. Não foram realizadas análises estatísticas para variáveis com baixa representatividade (origem gestacional e deficiência nutricional) e anemia relacionada a grupos específicos, como as de origem urogenital cuja etiologia é dependente da variável mulher em idade reprodutiva.

O estudo foi aprovado pelo Comitê de Ética em Pesquisa da Universidade do Oeste Paulista (protocolo no 586/2011).

\section{RESULTADOS}

Foram incluídos no estudo, 188 pacientes com diagnóstico de anemia ferropriva. A maior prevalência foi observada para o sexo feminino 128 (68\%). A origem etiológica mais frequente foi a perda sanguínea por via urogenital $71(37,7 \%)$, conforme observado na Tabela 1.

A condição de sexo e grupo populacional diferiu significativamente entre as origens etiológicas, o sexo masculino e o grupo de idosos demonstraram ser um fator de risco para a anemia de origem gastrointestinal baixa e alta. Para as perdas urogenitais não foi realizada a estatística entre sexo e grupo populacional por se tratar de um grupo formado por mulheres em idade reprodutiva e para as origens de menor representatividade numérica (gestacional e nutricional) não foi possível realizar a estatística. As estimativas foram calculadas por Odds-ratio (OR) e intervalo com $95 \%$ de confiança e estão sumarizadas na Tabela 2 . 
Tabela 1. Prevalência das etiologias da anemia ferropriva diagnosticadas em um hospital público do Estado de São Paulo ( $n=188$ ).

\begin{tabular}{lc}
\hline Origem Etiológica & Casos Registrados/Total de Casos (\%) \\
\hline Perdas urogenitais & $71 / 188(37,77)$ \\
\hline Menorragia & $69 / 71(97,2)$ \\
Câncer de útero & $1(1,4)$ \\
Perdas renais & $1(1,4)$ \\
\hline Perdas gastrointestinais altas & $65 / 188(34,57)$ \\
\hline Gastrite/ Ulcera Gástrica & $40 / 65(61,54)$ \\
Câncer gástrico & $10 / 65(15,38)$ \\
Gastrectomias & $7 / 65(10,77)$ \\
Varizes esofágicas & $4 / 65(6,15)$ \\
Polipose gástrica & $2 / 65(3,08)$ \\
Esofagite & $2 / 65(3,08)$ \\
\hline Perdas gastrointestinais baixas & $45 / 188(23,94)$ \\
\hline Doença diverticular & $14(31,11)$ \\
Câncer de cólon & $14(31,11)$ \\
Parasitose & $5(11,11)$ \\
Retite/colite & $4(8,89)$ \\
Angiodisplasia & $3(6,67)$ \\
Polipose intestinal & $3(6,67)$ \\
Hemorroidas & $2(4,44)$ \\
\hline Consumo aumentado/Gestacional & $5 / 188(2,66)$ \\
\hline Gestação & $5(100)$ \\
\hline Deficiências nutricionais & $2 / 188(1,06)$ \\
\hline Desnutrição & $1(50)$ \\
Vegetarianismo & $1(50)$ \\
Total Geral & $188 / 188(100)$ \\
\hline
\end{tabular}

A condição de sexo e grupo populacional diferiu significativamente entre as origens etiológicas, o sexo masculino e o grupo de idosos demonstraram ser um fator de risco para a anemia de origem gastrointestinal baixa e alta. Para as perdas urogenitais não foi realizada a estatística entre sexo e grupo populacional por se tratar de um grupo formado por mulheres em idade reprodutiva e para as origens de menor representatividade numérica (gestacional e nutricional) não foi possível realizar a estatística. As estimativas foram calculadas por Odds-ratio (OR) e intervalo com $95 \%$ de confiança e estão sumarizadas na Tabela 1. 
Tabela 2. Perfil epidemiológico dos pacientes com anemia ferropriva de acordo com as origens etiológicas com valor de significância (p), Odds-ratio (OR) e intervalos com 95\% (IC 95\%) de confiança $(n=188)$.

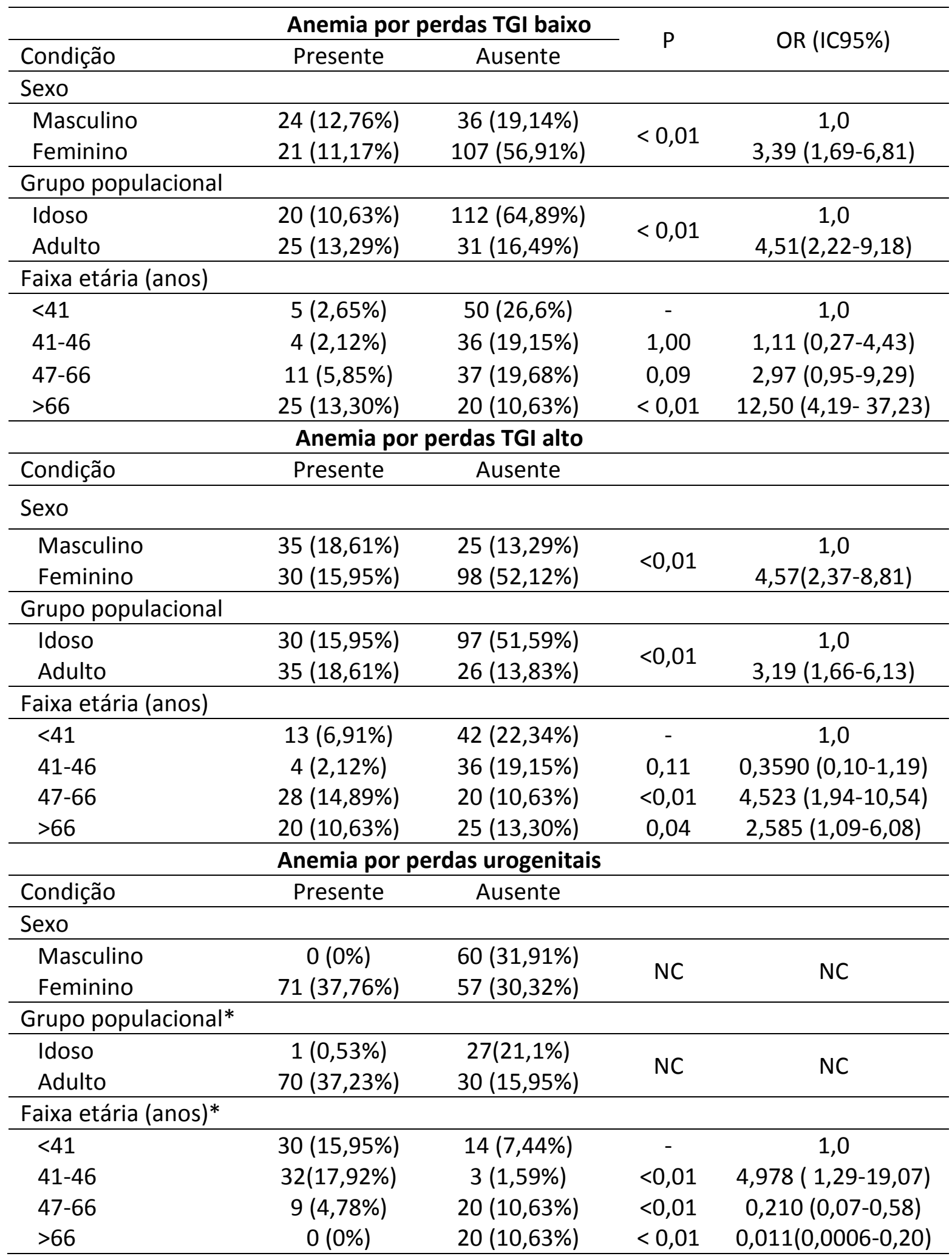

TGI - trato gastrointestinal; *análise realizada apenas para grupo relevante (mulheres). NC - Análise estatística não é valida para esta condição. 
DISCUSSÃO

Nas mulheres em idade reprodutiva, a anemia ferropriva se dá principalmente por perdas menstruais excessivas ${ }^{10}$, cuja prevalência pode chegar a $30 \%$ na América Latina $^{11}$. Neste estudo a origem urogenital foi responsável por $37,77 \%$ dentre todas as origens, sendo que a menorragia representou 97,2\% deste grupo (Tabela 1).

A hemorragia uterina disfuncional causa importante de menorragia, está associado com função ovariana anormal, anovulação, ou também pode ter origem endometrial. Outras causas ginecológicas que podem contribuir para as perdas vaginais irregulares e abundantes, tais como: pólipos, miomas uterinos e tumores pélvicos ${ }^{12}$. Apesar do trabalho não objetivar a pesquisa etiológica da menorragia, pôde-se observar nos prontuários a presença de mioma uterino em $60,8 \%$ dos casos.

O seguinte estudo realizado por Calabrish e $\mathrm{Katz}^{13}, 40 \%$ dos pacientes oncológicos apresentavam concentrações de hemoglobina menores do que $12 \mathrm{~g} / \mathrm{dL}$. Os pacientes com anemia ferropriva associada a alguma neoplasia foi encontrado em $1,4 \%$ na origem urogenital (câncer de útero), 15\% na gastrointestinal alta (câncer gástrico) e 31\% gastrointestinal baixa (câncer de cólon).

Detectou-se que $58 \%$ dos pacientes apresentavam sangramento de origem gastrointestinal, destes $34,7 \%$ perdas altas e
$23,94 \%$ baixas. O risco de ter anemia é estatisticamente maior em homens e no grupo de idosos, e concordante com Vannella et al. ${ }^{14}$ o risco aumenta progressivamente a partir dos 40 anos para as perdas altas (Tabela 2), o que mostra a necessidade de uma completa e rigorosa avaliação gastrointestinal para estes pacientes ${ }^{15}$.

Dentre as etiologias encontradas na origem gastrointestinal alta houve predomínio da gastropatia com 61,54\% (Tabela 1). Outra associação, altamente frequente na população, é a infecção por $H$. pylori que de acordo com Muhsen e Cohen ${ }^{16}$ aumenta o risco de anemia por deficiência de ferro em 2,8 vezes. A prevalência deste patógeno nos pacientes de etiologia gástrica foi de $32,5 \%$, dados que são inferiores ao encontrados por Santos et al. $^{17}$, cuja estatística foi de $63,4 \%$ encontrados em uma população em geral.

Outra etiologia encontrada na origem gastrointestinal alta é a gastrectomia que representa cerca de 10\% (Tabela 1), dados semelhantes ao encontrado por Avgerinos et al. ${ }^{18}$. Entre as complicações relacionadas à gastrectomia à Billroth I ou Billroth II e à cirurgia bariátrica, está a deficiência de ferro, resultando na anemia ferropriva ${ }^{19}$, que de acordo com Cançado e Chiattone ${ }^{20}$ a variação da incidência desta deficiência nos dois primeiros anos está entre 15 a $60 \%$ após o procedimento. 
As varizes esofágicas representam as veias colaterais porto-sistémicas mais relevantes ${ }^{7}$, neste estudo a incidência de pacientes com anemia ferropriva que apresentaram esta etiologia foi de $6,15 \%$. Os pólipos são geralmente assintomáticos. Os tipos mais comuns de pólipos gástricos são: as glândulas fúndicas, os pólipos hiperplásicos e os adenomas ${ }^{21}$. A polipose gástrica foi encontrada em 3,08\% dos pacientes com anemia ferropriva.

Em um estudo feito por Ruhl e Everhart $^{22}$ relacionando se há relação entre pacientes com esofagite e anemia ferropriva ou se é apenas uma coincidência, constatou que esta associação não está bem esclarecida e merece estudos mais aprofundados. Neste trabalho esta associação foi de 3,08\%.

Patterson e Johnston ${ }^{23}$ observaram a prevalência de 6,15\% de câncer gástrico em pacientes submetidos à investigação considerando apenas perdas pelo trato gastrointestinal, enquanto que neste trabalho foi observado em $15 \%$ dos pacientes com anemia de origem gastrointestinal alta.

Neste trabalho, a doença diverticular foi relacionada em $31 \%$ dos casos, câncer de cólon em $31 \%$, enquanto que a retite/ colite foi encontrada em $8,89 \%$. A polipose intestinal foi averiguada em $6,67 \%$, sendo esta encontrada na mesma proporção dos casos de angiodisplasia (6,67\%). Silva et al. ${ }^{24}$ realizou um estudo no Hospital Geral do Rio de Janeiro, onde os valores encontrados foram de $36 \%$ dos pacientes para a doença diverticular; $14 \%$ de câncer de cólon; 6,92\% de retite/colite; $23,5 \%$ de polipose intestinal e $4,6 \%$ dos casos eram de angiodisplasia. Sendo que a incidência entre os dois estudos foi aproximada, principalmente na doença diverticular, na retite/colite e na angiodisplasia.

As verminoses são infecções geralmente causadas, no Brasil, por Necator americanos, Ascaris duodenale, Schistossoma mansoni e Trichuris trichiura ${ }^{19}$. Qureshi et al. ${ }^{25}$ observaram presença de parasitose em $5 \%$ dos casos de anemia ferropriva, dados superiores aos nossos que em relação ao total foi de $2,65 \% \quad(n=5)$, muito provavelmente devido às adequadas condições sanitárias que o estado de São Paulo apresenta.

$\mathrm{O}$ aumento de necessidade do ferro está presente também na gestação, onde a anemia inicialmente é dilucional e em seguida ferropriva devido à alta demanda de ferro pelo feto ${ }^{26}$ que neste trabalho contemplamos com poucos casos $2,66 \%$ $(n=5)$ conforme observado na tabela 1. A prevalência de anemia ferropriva durante a gestação pode chegar a $50-70 \%$ das pacientes $^{27}$, embora provavelmente tenha ocorrido a subnotificação dos casos.

Sabe-se que o perfil da anemia ferropriva difere entre os países 
desenvolvidos e não desenvolvidos, e no Brasil a etiologia alimentar atualmente não se constitui frequente, pois além dos mecanismos de regulação na absorção do ferro $^{4}$, a alimentação é constituída $75 \%$ de ferro hêmico. Neste trabalho observou-se que $1,06 \%(n=2)$ dos casos de ferropenia eram por etiologia alimentar sendo, um paciente devido ao estilo de vida vegetariano exclusivo e outro, por inanição.

O perfil dos pacientes com anemia ferropriva que procuraram o serviço de hematologia, mostrou que a menorragia constitui a principal etiologia para mulheres em idade reprodutiva, representando cerca de um terço dos casos. Pôde-se observar que as origens etiológicas gastrointestinais, alta e baixa, representam juntas mais da metade dos casos e o risco de chances, para ambas, é maior para homens e idosos, sendo que para a origem relacionada ao seguimento alto 0 risco se torna estatisticamente maior a partir dos 40 anos. Sugere-se que, ao investigar a anemia ferropriva em mulheres em idade reprodutiva, deve-se inicialmente observar as causas uroginecológicas e, para homens pósandropausa, e mulheres pós-menopausa, pensar em causas gastrointestinais, considerando o risco aumentado para o sexo masculino.

\section{Conflito de Interesses}

Os autores declaram não haver qualquer potencial conflito de interesse que possa interferir na imparcialidade deste trabalho científico.

\section{REFERÊNCIAS}

1. Grotto HZW. Metabolismo do ferro: uma revisão sobre os principais mecanismos envolvidos em sua homeostase. Rev Bras Hematol Hemoter. 2008;30(5):390-7. DOI: http://dx.doi.org/10.1590/S151684842008000500012

2. Devuyst O, Jouret F, Auzanneau C, Courtoy PJ. Chloride channels and endocytosis: new insights from Dent's disease and ClC-5 knockout mice. Nephron Physiol. 2005;99:6973.

DOI: http://dx.doi.org/10.1159/000083210

3. Nemeth E, Ganz T. Hepcidin and ironloading anemias. Haematologica. 2006;91:727-732.

4. Falcão RP, Calado RT. Anemia Ferropriva. In: Zago MA; Falcão RP; Pasquini R editores. Hematologia: Fundamentos e Prática. 1.ed. São Paulo: Atheneu; 2004. p.223-229.

5. Goddard AF, Mcintyre AS, Scott BB. Guidelines for the management of iron deficiency anaemia. British Society of Gastroenterology. Gut. 2000;46(Suppl 34):IV1-IV5.

http://dx.doi.org/10.1136/gut.46.suppl 4.iv1

6. Batista Filho M, Souza Al, Bresani CC. Anemia as a public health problem: the current situation. Ciênc Saúde Col. 2008;13(6):1917-1922. DOI: http://dx.doi.org/10.1590/S1413$\underline{81232008000600027}$

7. World Health Organization. Worldwide prevalence of anaemia 1993-2005. Spain: WHO, 2008. 
8. Campos MGV, Figueiredo MS. Anemia Ferropriva. In: Figueiredo MS, Kerbauy J, Lourenço DM. (Coords.). Guia de Medicina Ambulatorial e Hospitalar da UNIFESP-EPM: Hematologia. 1.ed. Barueri: Manole; 2011. p.17-25.

9. Munõz M, Villar I, Garcia-Erce JA. An update on iron physiology. World J Gastroenterol. 2009;15:4617-26. DOI: http://dx.doi.org/10.3748/wjg.15.4617

10. Johnson-Wimbley TD; Graham DY Diagnosis and management of iron deficiency anemia in the 21st century. Ther Adv Gastroenterol. 2011;4(3):177-84 DOI: http://dx.doi.org/10.1177/1756283X1139873 $\underline{6}$

11. Rodrigues L, Jorge SRF. Deficiência de ferro na mulher adulta. Rev Bras Hematol Hemoter. 2010;32(Supl.2):49-5. DOI: http://dx.doi.org/10.1590/S1516$\underline{84842010005000059}$

12. Ballard L, Lyon DS, Jones JL. Inpatients with menometrorrhagia: etiologies, treatments and outcomes. South Med J. 2000;93(6):571-4.

13. Calabrich AFC, Katz A. Deficiência de ferro no paciente com câncer. Rev Bras Hematol Hemoter. 2010;32(supl.2):95-8. DOI: http://dx.doi.org/10.1590/S1516-

\section{9}

14. Vannella L, Aloe Spiriti MA, Di Giulio E, Lahner E, Corleto VD, Monarca B, Delle Fave G, Annibale B. Upper and lower gastrointestinal causes of iron deficiency anemia in elderly compared with adult outpatients. Minerva Gastroenterol Dietol. 2010;56(4):397-404.

15. Yun GW, Yang YJ, Song IC, Park KU, Baek SW, Yun HJ, Kim S, Jo DY, Lee HJ. A Prospective Evaluation of Adult Men with Iron-deficiency Anemia in Korea. Intern Med. 2011;50(13):1371-5. http://dx.doi.org/10.2169/internalmedicine.5 $\underline{0.5289}$

16. Muhsen K, Cohen D. Helicobacter pylori infection and iron stores: a systematic review and meta-analysis. Helicobacter. 2008;13(5):323-340. DOI: http://dx.doi.org/10.1590/S1516$\underline{84842010005000063}$

17. Santos IS, Boccio J, Santos AS, Valle NCJ, Halal CS, Bachilli MC, Lopes RD. Prevalence of Helicobacter pylori infection and associated factors among adults in Southern Brazil: a population-based cross-sectional study. BMC Public Health. 2005;5:118. DOI: http://dx.doi.org/10.1186/1471-2458-5-118

18. Avgerinos DV, Llaguna $\mathrm{OH}$, Seigerman $\mathrm{M}$, Lefkowitz AJ, Leitman $M$. Incidence and risk factors for the development of anemia following gastric bypass surgery. World J Gastroenterol 2010;16(15): 1867-70. DOI: http://dx.doi.org/10.3748/wig.v16.i15.1867

19. Cançado RD, Chiattone CS. Anemia ferropênica no adulto - causas, diagnóstico e tratamento. Rev Bras Hematol Hemoter. 2010;32(3):240-6.

DOI: http://dx.doi.org/10.1590/S1516$\underline{84842010005000075}$

20. Traina F. Deficiência de ferro no paciente submetido à ressecção gástrica ou intestinal: prevalência, causas, repercussões clínicas, abordagem diagnóstica e prevenção. Rev Bras Hematol Hemoter. 2010;32(suppl.2):7883. DOI: http://dx.doi.org/10.1590/S151684842010005000071

21. Goddard AF, Badreldin R, Pritchard DM, Walker MM, Warren B. British Society of Gastroenterology: The management of gastric polyps. Gut. 2010;59:1270-6. DOI: http://dx.doi.org/10.1136/gut.2009.182089

22. Ruhl CE, Everhart JE. Relationship of irondeficiency anemia with esophagitis and hiatal hernia: hospital findings from a prospective, 
population-based study. Am J Gastroenterol. 2001;96(2):322-6.

DOI:

http://dx.doi.org/10.1111/j.1572-

$\underline{0241.2001 .03513 . x}$

23. Patterson RN, Johnston SD. Iron deficiency anaemia: are the British Society of Gastroenterology guidelines being adhered to? Postgrad Med J. 2003;79:226-8. DOI: http://dx.doi.org/10.1136/pmj.79.930.226

24. Silva EJ, Câmara MAR, Gaidão E, Almeida EC. Colonoscopia: Análise crítica de sua indicação. Rev Bras Coloproct. 2003;23(2):7781.

25. Qureshi MIM, Khan SA, Farzana K, Murtaza G, Azhar S, Noreen S, Sherazi STA, Mumtaz A, Shah SH, Malik A, Ali N, Rasheed R, Razzaq R, Asad MHHB. Prevalence of iron deficiency in adult population: A case study from Khyber Pakhtunkhwa (KPK), Pakistan. Int J Phys Sci. 2012;7(11):1874-7. DOI: http://dx.doi.org/10.5897/IJPS12.020

26. Barón MA, Solano L, Peña E, Sánchez A, Del Real S. Estado de las reservas de hierro al inicio Del embarazo. Invest Clín. 2005;46(2):121-30.

27. Irshad G, Kousar S, Jafri SA, Ali I. Iron deficiency anemia significance of serum ferritin in diagnosis in pregnant females of Pakistan. Prof Med J. 2011;18: 475-478.

Recebido para publicação em 11/05/2013

Revisado em 13/04/2014

Aceito em 24/07/2014 